\title{
The Cabinet of Youth
}

\section{Chloe Mayne}

i don't feel as though i laugh so much anymore.

have i lost my spark? i implored.

has it scuttled inside the glass cabinet of youth

where relics, faded photographs and

other when-i-was-your-age paraphernalia are stored?

i telephoned the locksmith

and he informed me that

the only way to unlock a glass cabinet of that sort

is to take off one's shoes and socks,

run into the backyard on a rainy day

and roll most wildly in the mud -

the key is in there somewhere

if you feel about for long enough. 